\title{
AUSTRALASIAN JOURNAL OF PARAMEDICINE \\ Exploring paramedic communication and emotional expression in the workplace after responding to emergency calls
}

Cheryl Drewitz-Chesney BScN, MPH is Chapter Coordinator ${ }^{1}$

\section{Affiliation:}

${ }^{1}$ Rural and Remote Division of Family Practice, Canada and the University of Edinburgh, United Kingdom

https://doi.org/10.33151/ajp.16.714

\section{Abstract}

\section{Introduction}

Paramedics are at risk of developing mental distress and disorders when exposed to traumatic calls. Several studies demonstrate that when individuals are exposed to a traumatic event, there is a correlation between a lack of social support and emotional expression, and the development of disorders such as post-traumatic stress disorder and depression. Unfortunately, little is known about the social support that paramedics receive from their peers, particularly in the workplace after responding to traumatic calls.

\section{Methods}

The study aim was to learn about peer communication and emotional expression between paramedics in the workplace, after they respond to calls. This qualitative study was guided by constructivist, grounded theory. Semi-structured interviews were conducted with eight paramedics who worked for British Columbia Emergency Health Services in Canada.

\section{Results}

Two components of communication were described by participants: the content and logistics of peer communication. Content comprised of three types of peer communication: positive, negative and neutral, with the latter two potentially harmful to paramedics. Logistically, participants desired time to speak privately in the ambulance after calls, preferably with their regular partner or a peer with traits easing communication. These traits were identified as being comfortable with, trusting, sharing similarities and knowing one's partner.

\section{Conclusion}

Employers should facilitate positive peer communication among paramedics while considering the communication preferences described in this study. This could help to reduce rates of mental distress and disorders in paramedics.

Keywords:

paramedic or emergency medical technician; communication; emotion; social support; posttraumatic stress disorder (PTSD); depression 


\section{Introduction}

Paramedics are dispatched to calls to provide medical treatment for a range of illnesses and injuries (1). When a paramedic experiences a critical incident (Cl) from a call, or a cumulative effect of many $\mathrm{Cls}$, they have significant distress which can result in psychologically negative outcomes (2). A paramedic's risk of developing mental distress or disorders is increased by exposure to $\mathrm{Cls}$, chronic workplace stressors such as shift work, and a lack of support in the workplace $(3,4)$.

The prevalence of mental distress and disorders are higher among paramedics than civilians $(5,6)$. Among paramedics, mental health stress leave is common $(7,8), 15$ to $30 \%$ experience depression $(7,9)$, and 11 to $25 \%$ develop posttraumatic stress disorder (PTSD) $(5,7,9)$. A survey $(\mathrm{N}=5148)$ comparing Canadian police, firefighters, paramedics, dispatchers and corrections officers reported that paramedics had the highest rate of lifetime suicidal ideation at $41.1 \%$ (10). Recently, more studies have examined PTSD in military members and first responders, who include police, firefighters and paramedics, including quantifying the prevalence of PTSD and identifying coping methods and treatment; however, there has been less attention on preventing mental disorders in paramedics (11-13).

Although few studies include paramedics, several studies have explored preventive and protective factors from PTSD among military, first responder and civilian populations. Among individuals exposed to trauma, an association has been found between having a mental disorder(s) and suppressing feelings and/or having difficulty expressing emotion, and receiving little social support (13-17). One study of police $(\mathrm{N}=83$ ) reported that one of the strongest predictors and protectors from PTSD and post-traumatic stress symptoms (PTSS) was when the police received peer support during or immediately after a traumatic event (18). In a study of civilian adults ( $\mathrm{N}=1027)$ who were exposed to the same traumatic event, individuals with PTSS far more frequently did not share nor wanted to share emotion compared to individuals without PTSS (19). Two meta-analyses that included studies with military and civilian populations concluded that low levels of social support after a trauma correlate with the development of PTSD or PTSS $(20,21)$.

While both social support and emotional expression can help prevent first responders from developing mental disorders, not all social support and responses to expressing emotion are equally preventive $(19,22)$. A meta-analysis evaluating social support and mental health among first responders found a larger effect size for perceived social support than received social support (22). There are differing theories as to how perceived social support helps prevent mental distress, including improving resilience and processing trauma (22). Additionally, there are different forms of social support, including positive social support, which can reduce fear responses following trauma, and negative social support, which can exacerbate those responses (23).

During emotional expression, the affected person openly talks with another person about the event causing their emotional response, as well as their feelings and reactions (19). Like social support, this can elicit different responses. When the other person is receptive to the sharer's emotional expression, the former may respond with one or both of the following responses: socio-cognitive, which considers the sharer's perspective and involves reasoning their beliefs and thoughts (24) and a socio-affective response, which is empathetic and often makes the sharer feel supported, comforted, consoled and validated (19). Among studies ( $\mathrm{N}=8$ ) that examined the timing of sharing emotion, the majority of participants shared their feelings about the event the day it occurred (19). Additionally, peer support immediately following Cls was found to be most effective in preventing PTSD in police (17) and relieving stress among paramedics (25).

Although informal social support among first responders has been shown to significantly contribute to their mental wellbeing after being exposed to trauma (22) no studies were found describing how paramedics perceive social support and express emotion with peers at work after calls. Paramedic communication and emotional expression that occurs after calls was explored since immediately after the trauma is when most individuals sharing emotion first express their feelings and this time may be most beneficial to paramedic mental health $(19,25)$.

\section{Method}

The primary research question was: How do paramedics describe workplace communication and emotional expression with other paramedics, after responding to calls? To explore the social context of communication and emotional expression, additional sub-questions were developed: How do paramedics feel about communicating with peers? What do paramedics believe influences their communication and emotional expression with peers? Themes that emerged from the primary question and first sub-question include with whom, where, when and how paramedics communicate, and traits enhancing peer communication. The study was guided by constructivist grounded theory (GT). Constructivist GT involves an iterative process of comparing data for differences, similarities and themes, and considers the meaning and understanding of the topic to be co-constructed between the researcher and participant(s) (26). The framework used was social theory, which explores how social interactions and group behaviour impact our actions and perspectives (27).

\section{Sampling}

Participants were employees of British Columbia Emergency Health Services (BCEHS), a provincial emergency health 
Cheryl Drewitz-Chesney: Communication between paramedics after emergency calls

service in Canada that employs over 3600 paramedics in urban and rural settings (28). Participants were primary care paramedics (PCPs) or advanced care paramedics (ACPs), worked in a ground ambulance with another paramedic, had not taken a course specifically about peer communication or critical incident stress (CIS) while training to become a paramedic, worked as a paramedic for at least 1 year and had access to an electronic device with Skype.

Sampling five to eight participants permits ample time for completing a detailed data analysis (29). Eight paramedics were recruited from private Facebook groups established for BCEHS paramedics. Data saturation guided sampling and was reached in over-arching themes after the sixth participant. Sampling was also based on 'information power' which suggests that when sufficient information is gleaned from participants, a smaller sample size is required (30). Sufficient information power for this study was derived from in-depth interviews and analysis, a focussed study aim, clear dialogue between the researcher and participants, and use of an existing theoretical framework.

Participants were sampled using convenience, then purposive sampling. Convenience sampling enabled initial recruitment. Snowball and quota sampling were the two forms of purposive sampling used. Participant demographics are outlined in Table 1.

\section{Data collection}

An in-depth, semi-structured interview was conducted with each participant (50 to 90 minutes each). Questions evolved to explore emerging themes. Interview questions are listed in
Table 2.

Interviews were conducted using Skype, and participants could choose to interview via video $(n=5)$ or audio only (31). Each interview was transcribed verbatim. Each transcript was read at least three times to improve accuracy and familiarity (32).

\section{Data analysis}

Data analysis followed an iterative process of coding, memoing and reflexivity. Coding comprised two phases: initial line-by-line coding, then focussed coding (33).

Atlas.ti was utilised to record and organise codes and memos and create diagrams. Memoing included recording thoughts and ideas that emerged during the research process (34). Rigor was achieved through credibility, transferability, dependability and reflexivity (35). Credibility was enhanced through triangulation, including interviewing the manager of the BCEHS CIS program, Marsha McCall, and Anonymous, a retired BCEHS paramedic, whom substantiated some participant data (36). Thick descriptions and a diverse sample contributed to credibility and transferability. An audit trail was maintained throughout the research process which enhanced dependability (36). Journaling and bracketing enriched reflexivity $(37,38)$.

\section{Insider status}

The recruitment message stated the interviewer previously worked as a paramedic. This may have improved access to participants (39). Although being an insider can lead to role

Table 1. Participant demographics

\begin{tabular}{|c|c|c|c|c|c|c|}
\hline $\begin{array}{c}\text { Participant } \\
\text { pseudonym }\end{array}$ & $\begin{array}{c}\text { Gender: } \\
\text { Male: (M) } \\
\text { Female: (F) }\end{array}$ & Age* (years) & $\begin{array}{c}\text { Level of } \\
\text { training }\end{array}$ & $\begin{array}{c}\text { Years of } \\
\text { experience }\end{array}$ & $\begin{array}{c}\text { Area(s) worked } \\
\text { during career:\# } \\
\text { Metro (M) } \\
\text { Rural (R) }\end{array}$ & $\begin{array}{c}\text { Type of partner(s) } \\
\text { during career } \\
\text { Regular (Reg) } \\
\text { Different (D) }\end{array}$ \\
\hline Krista & $\mathrm{F}$ & $25-39$ & PCP & $1-5$ & $\mathrm{M}+\mathrm{R}$ & $\mathrm{D}$ \\
\hline Tanya & $\mathrm{F}$ & $25-39$ & $\mathrm{PCP}$ & $6-10$ & $\mathrm{M}+\mathrm{R}$ & $\mathrm{D}$ \\
\hline Hannah & $\mathrm{F}$ & $40-54$ & PCP & $11-15$ & $\mathrm{M}+\mathrm{R}$ & $\mathrm{D}$ \\
\hline Dennis & $\mathrm{M}$ & $25-39$ & $\mathrm{ACP}$ & $11-15$ & $\mathrm{M}+\mathrm{R}$ & Reg + D \\
\hline Sean & $\mathrm{M}$ & $55-69$ & PCP & $26-30$ & $\mathrm{M}+\mathrm{R}$ & Reg + D \\
\hline James & $\mathrm{M}$ & $40-54$ & ACP & $16-20$ & $\mathrm{M}+\mathrm{R}$ & Reg + D \\
\hline Dave & $\mathrm{M}$ & $25-39$ & PCP & $6-10$ & $\mathrm{R}$ & $\mathrm{D}$ \\
\hline Linda & $\mathrm{F}$ & $40-54$ & $\mathrm{ACP}$ & $16-20$ & $\mathrm{M}$ & Reg + D \\
\hline
\end{tabular}

${ }^{*}$ The exact age and years of experience for each participant is not listed as there is a possibility that information, coupled with the other demographics, could reveal their identity. Instead, broader categories were developed for age and years of experience. \#Metro stations are located in Metro Vancouver and southern Vancouver Island where there is a high concentration of people. Rural stations are located in areas ranging from few people to $>120,000$.

${ }^{\wedge}$ Area(s) worked during career and Type(s) of partner during career list whether participants have worked metro or rural and if they have had regular or different partners at any time throughout their careers. These are important to document as participants shared that paramedics may experience different peer communication based on where they work and their type of partner. However, listing each participant's current work location and type of partner could make some identifiable. 
Cheryl Drewitz-Chesney: Communication between paramedics after emergency calls

confusion and bias (40) the author worked as a paramedic more than 10 years ago and never for BCEHS or with the participants.

\section{Ethics}

This study received ethical approval from the University of Edinburgh's Usher Research Ethics Group. Each participant provided verbal and written consent.

To minimise the risk of psychological impact, participants were never asked to recall specific calls or details. Participants were monitored for signs of distress during each interview (none were noted). BCEHS paramedics have access to three services offering support and counselling. These services were listed on their information letters. At the conclusion of each interview, participants were asked if they wanted a referral to any of the services, which all participants declined.

\section{Results}

Two of the overarching themes that emerged were: (a) logistics and content of communication, and (b) traits that ease communication. 'Peer communication' herein refers to discussions between work partners after a call, unless otherwise specified.

\section{Logistics of peer communication}

Participants described the logistics of discussing calls, including with whom, where and when they prefer to talk. They expressed a preference for discussing calls with their partner and stressed that partners understand call circumstances and are less likely to judge treatment decisions. 'It is the person who was with you during the call and who knows what happened and who knows the circumstances' (Linda).

Specifically, participants preferred discussing calls with their

\section{Table 2. Interview questions *}

\section{What does working as a paramedic mean to you?}

2. How do you feel after responding to calls?

3. How do you express your feelings with other paramedics after emergency calls?

4. How do you feel colleagues expect you to communicate and behave after calls?

5. Can you describe typical communication with your partner following a call?

6. Why do you believe paramedics communicate like this with each other?

7. Does this change from location to location? ie. the hospital, ambulance or station

8. Is communication with your partner different when the environment changes? ie. other people around, supervisor present, personal connection to call

9. Are there certain calls or subjects that are easier to talk about with some paramedics than others?

a. Can you tell me about the paramedics that are easier to talk to? The easier calls?

b. Can you tell me about the paramedics that are harder to talk to? The harder calls?

10. How do your partners communicate with you at work following calls?

11. Can you tell me how you respond to your partners talking about calls?

12. Some people believe that the more exposure to bad calls a paramedic has, the more support they'll receive. Can you describe your thoughts about this?

13. Can you tell me how you would describe communication between paramedic partners to people that don't work in EMS?

14. If your partner appears distraught after a call i.e. crying or verbalises fear, how does that make you feel?

15. How does that make you feel about them or their ability to do the job?

16. Describe how peers react when you discuss your thoughts about a call (If the participant discusses their feelings with peers asking question is dependent on how participant answers question \#5)

17. How does this make you feel?

18. Can you describe your ideal communication at work following emergency calls with peers?

19. Are there barriers preventing you from your ideal peer communication? If the participant says there are barriers: Can you tell me about those barriers?

20. What do you believe could improve paramedic communication?

21. Has your communication with peers changed since you first became a paramedic?

22. If the paramedic reports communication has changed: Could I ask you how your communication has changed with peers?

23. Is there any advice you'd like to give to new paramedics about communicating with co-workers following emergency calls?

24. Is there something else you think I should know to better understand paramedic communication?

25. Is there anything you'd like to add?

*No participant was asked every question. Instead, questions were asked based on the experiences the participant shared with the author and themes that required further exploration 
regular partner. Regular partners can more readily identify stressors, advocate for each other and encourage discussion of calls. James recommended when possible, to rely on regular partners: '... for those of us that have regular partners, regular stations, rely on your partner... They're gonna know if something's up with you.' Paramedics working with different partners may be unable to recognise changes as readily as they would in a regular partner. When paramedics are without a regular partner or are uncomfortable speaking with their current partner, they often don't discuss calls. Instead, some participants said they speak with long-time paramedic friends or coworkers with whom they have similarities, ie. age or training level.

Paramedics have three primary options of where to communicate at work: their station, the hospital(s) and ambulance. They seem to rarely discuss calls at the station, particularly when other paramedics are present. Crews that did not attend the call may be critical of patient treatment or insensitive to what peers witnessed. 'Whenever I talk at the station it's a little more guarded because there's always a chance of someone being around' (Dennis). If a peer joins their conversation, Dennis and his regular partner change the topic. Some also felt an expectation among experienced peers to suppress their feelings: '.. [paramedics] may not feel comfortable talking about it just because they don't want to hear the response from some old crony who's going to tell them to suck it up' (Dave).

Others may restrict talking at the station in order to protect themselves and/or their peers. Linda, an experienced ACP, avoids debriefing in the station so she doesn't have to censor talk around PCPs: 'You've gotta be very careful and sensitive to, you know, everyone's there to do a job and everyone's job is a little different... When you're debriefing you don't want to worry about how someone's feeling.'

Participants limited conversation at the hospital to technical discussion (see positive communication). Some participants were concerned their peers and the public might hear their conversations. Generally, they used hospital time to clean equipment and finish paperwork.

Of the three locations, the privacy of talking in or outside of the ambulance without coworkers nearby was the preferred space for discussing calls. Participants said they usually spoke in the ambulance when leaving the hospital. 'Most of the debrief happens in the car [ambulance]. It's a closed environment. It's just you and your partner' (Linda).

'In the ambulance seems to be the place where most intimate conversations will take place about how people feel, and that's usually just two people, right? So it's limited in opinion and we're usually the two people that were there...' (Tanya).

If Linda and her partner are still discussing the call when they arrive at the station, she puts the ambulance in park and they continue talking in the privacy of the ambulance.

Speaking in the ambulance after leaving the hospital aligns with when most participants prefer to talk: immediately after calls. 'I think it's good to talk about it right away' (Dave). Unfortunately, paramedics often do not have adequate time to talk. Several participants expressed their frustration over 'calls waiting'. These calls are waiting to be dispatched as no crew is available. When BCEHS paramedics offload their patient(s) at the hospital, dispatch can immediately send them to a waiting call. This can limit paramedics' ability to discuss Cls. James desired: ' ...to be given the time uninterrupted, unbothered... Give us our time to decompress'.

\section{Content of peer communication}

Participants described three categories of peer communication: positive, negative and neutral.

\section{Positive communication}

Positive communication helps paramedics to process and feel better about Cls. Participants frequently discuss technical components first, then their emotions. Occasionally, humour is used to initiate conversation.

All participants said they discuss technical components, defined as the medical aspects of a call including patient pathophysiology and medical treatment: "What we did. What we saw. If [treatment] was appropriate. If there's things we could have done better. What went wrong? What went right?' (Linda). Many participants said they also review their actions on the call with their partner: 'I just ask them how they think the call went. What could we do different? Did we miss anything?' (Dave).

A benefit of technical communication is that it does not require disclosing one's feelings. Expressing emotion can be difficult for paramedics when they are unsure how their partner will respond. Because of this, some participants said they exclusively discuss technical components when working with paramedics they don't know well. Krista, who works with different partners, said: 'When I talk about calls with my partner-of-the-day, I usually just talk about the technical aspects of the call... unless I know the person... I don't usually talk about the emotional impact of the call'. Discussing technical components may allow paramedics working with different partners to engage in some positive communication, and could be a socio-cognitive response to a peer discussing a traumatic call (24).

The second component of positive communication is expressing emotion, which includes sharing one's feelings and checking-in with peers. Dennis explained the importance of sharing feelings after a call: 'The more you can make it a habit, the more aware you can become of what's happening and how you're feeling about it, the more you can be aware of when things go wrong'. When individuals socially share emotions and 
receive a positive response, feelings of self-doubt, vulnerability and isolation are often alleviated, and they experience a sensation of relief (19).

Participants also described checking-in on peers, which involves assessing and asking how their partner or peer is doing. Checking-in can occur during the shift or sometimes with partners days later by phone or text. This suggests that paramedics with a regular partner could receive more support as regular partners have more opportunity to check-in at work than paramedics working with different partners each shift.

Some participants felt a responsibility to check-in with paramedics who were quiet or appeared distraught after a call, even if that was not their regular partner: '... you can kind of see their actions and their body language have changed and so then it's for me to provoke with a question like "Are you okay after that?"' (Tanya). Paramedics who provide empathetic support to peers sharing emotion may be demonstrating a socio-affective response (19). Some were encouraged when they learned of the positive impact their check-in made. It was significant for Sean when a peer expressed how impactful his check-in was: '...for me to know that that one text message made such a big difference when he was in his moment of crisis, to me it's really reassuring...'

Several participants described feeling nervous about checkingin with co-workers, particularly with older, experienced, male paramedics, some of whom have mocked others for asking about feelings. However, concerns over receiving a negative response may be superseded when paramedics feel they improved or even saved a peer's life.

When participants receive check-ins they seem to feel relief and reassurance that other paramedics are also impacted. When asked how it makes her feel when a peer checks-in, Hannah replied: '...I think it's good because if somebody says that to me, then they obviously acknowledged that it was upsetting, and it was probably upsetting to them too...'. Feeling legitimised and recognised is another outcome of socio-affective responses (19). Although participants said peers check-in on them, many felt that they check-in more than their peers do. This could be an example of selection bias where the paramedics sampled are more open to communicating than others.

Lastly, paramedics may use humour as it is a more accepted and an indirect method of expressing emotion among first responders. Of note is that all participants who identified humour as positive communication worked with different partners. Paramedics may use humour to assess if unfamiliar peers are open to talking. This is similar to police who use humour to lessen the negative impact of calls and to express their emotions in a socially acceptable way, ie. laughing at, rather than appearing affected by, calls (41). Unfortunately, if neither paramedic engaging in humour is willing or comfortable expressing emotion, humour could be used to hide emotion and their communication could remain neutral or even become negative (42).

\section{Negative communication}

Although described as the least common form of communication, all participants had received negative responses from peers after calls. After mentioning a call was upsetting, Dave, was told by a co-worker: 'That's part of the job. You signed up for it, you just have to learn to deal with it'. Krista recalled a partner laughing about a new paramedic who was upset over the death of an elderly woman. She was told: '... that's so ridiculous. You need to harden up if you want to do this job, and old people die'. In addition to mocking one's emotional reaction to a call, some paramedics criticise their peers' actions. Sean explained that paramedics who do not attend the call may critique a crew for the medical treatment provided. Consequently, paramedics may be afraid of having their actions judged and restrict their discussion around certain peers. Although several participants stressed negative responses are rare, they seem significant enough to force paramedics to adjust their behaviour. 'I'm a little bit more careful about what I say at the start of the shift before l've gotten a good handle of who my partner is' (Krista). Krista said she refuses to work with paramedics known for negative communication and that her friends warn each other not to work with those co-workers. Others said they attempt to disregard negative responses by looking at their phones or not responding to hurtful comments.

\section{Neutral communication}

A neutral response can include consciously downplaying calls or not discussing them. Neutral responses are so common that Hannah, an experienced PCP, does not expect to talk about calls. Participants described common ways paramedics downplay calls: acting bored, displaying bravado and laughing. Linda spoke of acting 'bored': 'So how you portray yourself after [the call] is you know, dust it off. It's almost bored, like "oh my God, that was so boring. I had that. No problem"'. When several crews are present at a busier station, bravado is commonly displayed until paramedics are one on one. '... in metro, there's a lot more...bravado and joking around about things...If you can get one on one with someone, they're usually a lot more receptive and a lot more empathetic' (Dennis). When paramedics do not transition from using humour to positively discussing calls, humour can be used to mask feelings (43). Paramedics seemingly act bored, show bravado and laugh about calls in an attempt to appear unbothered by them.

When paramedics work with an unknown partner or are unsure how their peer(s) will respond to discussing calls, many suppress their emotions and avoid talking about calls. 'You've literally just met this person fifteen minutes ago. I mean it's obviously more of a barrier to talk to them' (Krista). Unfortunately, neutral responses can be harmful when they prevent paramedics from processing calls. 
Cheryl Drewitz-Chesney: Communication between paramedics after emergency calls Australasian Journal of Paramedicine: 2019;16

\section{Factors easing peer communication}

Four factors appear to make peer communication easier for paramedics: comfortable being vulnerable, trusting, sharing similarities and knowing one's partner.

\section{Comfortable being vulnerable}

Participants stated they need to feel comfortable with their peer in order to talk to them. They described feeling comfortable when they could be vulnerable with each other: '... having a mutual sense of your vulnerability is safe with each other' (Sean). Participants described being guarded around peers they're uncomfortable with, as they're unsure how they'll be received if they show emotion.

Linda described two additional barriers to feeling comfortable being vulnerable: machoism and ego. A macho atmosphere can prevent some paramedics from sharing their feelings (44). Tanya described a fear of appearing weak and affected by calls:

'A lot of it is for fear of judgement, for fear of being unnecessarily sensitive or judgement of being weak. There's a lot of pride involved and just thinking nope I can do this, I'm better than this, I'm above this'.

\section{Trust}

Trusting peers appears critical to paramedic communication: '...if [paramedics] trust the person they're with, they're more likely to open up and to talk, and if they don't have that trust... then they just shut absolutely down' (Sean). Some participants said trust forms when doing difficult calls together. This could be because trust can build when paramedics see each other's strengths and limitations, and ask for help (45). Paramedics who attend many calls together at busy stations or as regular partners, could develop trust faster.

When gauging if a peer is trustworthy, Linda said she considers if they're kind to patients and preserve patient confidentiality. Paramedics may correlate peers' actions towards patients with how they'll be treated.

\section{Similarities}

All participants said communication is easier with other paramedics when they share one or more of the following: family demographics, age, level of training, comparable call experiences and attending calls together. The latter three are discussed here.

Participants said when they work with a partner that has a different level of training, it is difficult to adequately discuss the call. Linda prefers talking with other ACPs:

'It's gotta be licence level equivalent for me because what I see in a call and what a partner who's not the same qualifications see[s], or my responsibility versus their responsibility are very different... I need to know that someone saw what I saw, knows what I know, would have done what I done [sic]'.
Discussing the call and feeling reassured the correct treatment was provided is challenging when one's partner does not fully understand the diagnosis or treatment because they have less training. When she works with PCPs, Linda said communication is usually restricted to questions about pathophysiology. This prevents her from reviewing technical and emotional aspects of the call. Hannah, a PCP, disclosed the same struggles when working with emergency medical responders (EMRs), who have less training than PCPs. Partners with the same level of training may be better able to understand each other's roles, responsibilities and concerns after a call. Dennis, an ACP said: 'It's a lot easier to... relate and connect to some of the different stressors as other [ACP] members'.

The lesser-trained partner also faces challenges. When she's working with ACPs, Hannah said of technical discussions: '...it's way above me'. The lesser-trained partner may feel uncertain if the treatment was correct or adequate and may rarely discuss emotions after the call. This finding may vary with different levels of training and organisational differences between provinces and countries.

Communication appears easier between similar paramedics. This may be exemplified in the CIS program which organises trained paramedics to phone and offer support to peers who respond to potentially traumatic calls. CIS program manager McCall (March 26, 2018) said some paramedics only want to speak with peers who are similar to them (eg. equal training or experience).

Similar call experiences also ease communication. '... if they have a similar experience background, they are usually easier to talk to about anything' (Dennis). Paramedics working in high call volume areas often know a peer(s) with similar call experiences as they are often exposed to more calls than paramedics working at rural stations located in smaller communities.

Lastly, many participants said they preferred talking to paramedics with whom they attend the call. 'It's just easier talking with somebody that was actually with you' (Hannah).

Intense shared emotional experiences generate camaraderie and may encourage paramedics to talk with their partner (45). 'It is the person who was with you during the call, and who knows what happened, and who knows the circumstances' (Linda).

\section{Knowing one's partner}

All participants emphasised the importance of knowing one's partner which included spending time together and having a regular partner. Spending time together allowed partners to learn each other's preferred method of patient treatment, their personality and perspective on peer communication. 'I think the 
Cheryl Drewitz-Chesney: Communication between paramedics after emergency calls

more time you spend with someone, the more you sort of suss out their personality... you sort of get a good feeling about what you can and can't talk to them about' (Dave). Those with regular partners usually spend considerable time together and are more likely to have positive communication. '... if I'm working with my regular partner, those communication lines are very, very open and we can talk about the tough calls, how we're feeling, if there's anything we would have done different [sic]' (Sean).

However, not all paramedics in regular partnerships can be assumed to have positive communication. Some do not trust or feel comfortable being vulnerable with their regular partner. Sean even warned of some hostile partnerships. Even those in a good partnership can experience poor communication when working with a different partner, ie. their regular partner is away. Dennis described talking about calls when he's not working with his regular partner: '... it's less of like a good debrief. It's more of a brief discussion with my partner-of-the-day'.

The benefits of having a regular partner with positive communication were not lost on participants working with different partners. 'I really envy the paramedics that have regular partners that they know, and they trust, and they can talk to' (Krista). When asked to describe his ideal communication, Dave said: '...I would have a regular partner and that partner would be someone who is just like family to me. We would just talk about everything, without even hesitating...'

All participants were asked 'Can you describe your ideal communication at work following emergency calls with peers?' Their answers are summarised in Table 3.

Being an open-ended question without specific prompts, it is notable how many participants desired time to talk, with the partner they attended the call, and ideally spoke with a peer having at least one of the traits easing communication.

\section{Discussion}

This study explored the content, logistics and traits easing paramedic peer communication. Content comprised of positive, negative and neutral communication. Positive communication seems to be more common between regular partners and with peers that have traits easing communication. It can include both socio-cognitive responses (ie. discussing technical components) and socio-affective responses to emotional expression (24).

Neutral communication was described more commonly between unfamiliar partners. Negative communication seems less common yet was experienced by all participants. Unfortunately, negative and even neutral peer responses can increase and sustain fear from a traumatic call (22). In fact, individuals receiving a neutral response to a traumatic event are twice as likely to have intrusive memories as those who receive negative responses (46). Abrasive negative responses may be easier to reject, however, when a paramedic receives '...no support or a neutral reaction [it] may be more invalidating long-term...' (46). This finding, coupled with the normalcy of neutral responses, suggests many paramedics may feel disregarded by their peers and don't receive adequate social support after $\mathrm{Cls}$. This could be a contributing factor to why $49.1 \%$ of Canadian paramedics $(\mathrm{N}=311$ ) screened positive for a mental disorder (7). Future research could aim to quantify not only how often paramedics receive positive, negative or neutral communication but how often they feel supported and/or invalidated by peers.

The logistics of peer communication include where, when and whom paramedics prefer to talk with after responding to calls. This may be the first study identifying that paramedics prefer the privacy of speaking in the ambulance after calls, with their partner. This is significant as many paramedics work as a twoperson crew in an ambulance.

Participants expressed frustration at being sent to 'calls waiting' immediately after transferring patient care to hospital staff. Although crews have time in the ambulance while driving to the next call, that time is generally used to prepare for the next patient (25). Responding to back-to-back calls limits paramedics' ability to discuss $\mathrm{Cls}$. Some paramedics experience lengthy offload delays which render them unable to talk privately after the call as they are caring for patients in hospital hallways. Nearly $60 \%$ of paramedics surveyed $(\mathrm{N}=813)$ from eight Western countries said they lacked time to talk after $\mathrm{Cls}$, which resulted in higher distress levels than among paramedics who felt they had adequate time (47). A previous study identified that a 30 to 60 minute break after $\mathrm{Cls}$ is important to paramedics and when they receive downtime after calls, rates of depression are reduced (25). The findings from this study may help to identify some aspects of positive communication that are helpful to paramedics during downtime.

Table 3. Participant's ideal communication

\begin{tabular}{|l|c|c|c|c|c|c|c|c|}
\hline & Krista & Tanya & Hannah & Dennis & Sean & James & Dave & Linda \\
\hline Time to talk & $\checkmark$ & $\checkmark$ & & $\checkmark$ & $\checkmark$ & $\checkmark$ & $\checkmark$ & $\checkmark$ \\
\hline At least one of the traits easing talk & $\checkmark$ & $\checkmark$ & $\checkmark$ & $\checkmark$ & & $\checkmark$ & $\checkmark$ & $\checkmark$ \\
\hline Talk with partner on call & $\checkmark$ & $\checkmark$ & $\checkmark$ & $\checkmark$ & $\checkmark$ & $\checkmark$ & $\checkmark$ & $\checkmark$ \\
\hline Learning patient outcome & & $\checkmark$ & & & & & & \\
\hline Supervisor support & & & & & $\checkmark$ & & $\checkmark$ & \\
\hline
\end{tabular}


Cheryl Drewitz-Chesney: Communication between paramedics after emergency calls

Australasian Journal of Paramedicine: 2019;16

Employers could implement a policy allowing crews to request some uninterrupted time after Cls. Unfortunately, due to existing workplace culture, paramedics may feel uncomfortable asking for time to talk. Alternatively, supervisors could enact a 30 to 60 minute break for crews after a potential $\mathrm{Cl}$, during which the crew is not permitted to be sent by dispatch to a call.

The few studies exploring paramedic peer support have typically defined "peers" as partners, coworkers and even supervisors $(3,47)$. This study demonstrated that participants were uncomfortable talking with just any co-worker, and preferred instead to speak privately with the paramedic with whom they attended the call, who was ideally their regular partner or at least had traits easing communication.

This study also highlights the benefits of, and paramedic preference for, working with a regular partner. Regular partnerships may help to reduce rates of mental distress. In many ambulance services, only experienced paramedics have seniority to bid into positions with a regular partner. Many part-time and/or on-call or casual paramedics work for years with different paramedics before working with a regular partner. In some emergency services such as BCEHS, the employee union influences the employers' staffing and deployment models. Employers should consider, and when applicable unions should advocate for, opportunities for new and part-time paramedics to have regular partners. Although regular positions cost more upfront, they could save employers disability and sick time costs by helping to facilitate positive communication and social support among paramedics.

Lastly, participants identified four traits that ease communication: feeling comfortable, trusting, sharing similarities and knowing one's partner. A study exploring police emotion reported that police also have an easier time speaking with coworkers they have similarities with and know (48). Identifying these traits could help to reduce PTSD in paramedics. Importantly, police who can easily discuss traumatic calls with peers experience fewer PTSS (49). The four traits easing communication could be incorporated into existing workplace programs. For example, CIS programs could match paramedics exposed to trauma with trained $\mathrm{CIS}$ peers that have similar experiences and training level to ease discussion between them.

\section{Future research}

Critical care paramedics were not included as they generally work in air ambulances (eg. helicopter) in British Columbia. This may present unique communication challenges that warrant study, including working with pilots. Further research should also explore when supplementary individuals (eg. students or observers) join a ground ambulance two-person crew. They may experience different communication, barriers and/or facilitators that impact both the student and paramedic(s).
This study identified several groups that could face greater communication challenges. These include new and part-time paramedics, rurally-stationed paramedics and partners with different levels of training. These groups should be further researched, including evaluating if paramedics who work with regular partners experience less stress and/or mental disorders than paramedics who work with different partners. Some workplace interventions are suggested in this article though more research, evolving workplace culture and new policies are needed to support positive communication.

Negative or deviant cases can help to provide a balanced perspective and improve rigor (50). Many participants described negative communication from older, experienced paramedics, though none described their own responses as negative. The low likelihood that paramedics regularly engaging in neutral or negative communication would participate in a communication study may have contributed to having no deviant case.

\section{Limitations}

Limitations of this study include a sample size of eight participants. Findings are unlikely to be representative of all paramedics in British Columbia, Canada or other developed countries. However, thick descriptions, participant demographics and organisational details of BCEHS are provided so readers may assess how findings transfer to other settings.

Several types of paramedics that were not sampled include supervisors, EMRs and critical care paramedics. Some supervisors respond to calls; their post-call communication should be studied as they generally work alone. EMRs were not included in this sample as they are not licenced in many Canadian provinces and may have unique communication experiences that are inapplicable to other paramedics. However, as EMRs work in rural and remote areas in British Colombia and are often new employees, they may be at greater risk of not receiving positive communication. This study also contains a risk of self-selection bias. Participants may have been more open to, desired, or placed more importance on peer communication than their peers (51). To account for this possible bias, participants were also asked how their peers communicate.

\section{Conclusion}

This study appears to be the first identifying the components and logistics of paramedic peer communication. Participants desired adequate time after calls to have positive communication in the ambulance where they preferred to speak with their regular partner or a peer with traits easing communication. Workplace culture, programs and policies must 
begin to encourage and facilitate positive communication and social support among paramedics, and address factors increasing the risk of receiving neutral and/or negative peer responses. Increasing positive communication and encouraging emotional expression among paramedics could help reduce their high rates of mental distress and disorders.

\section{Conflict of interest}

The author of this paper reports no competing interests. The author of this paper has completed the ICMJE conflict of interest statement.

\section{Acknowledgements}

The author wishes to thank the paramedics who participated in this study, and Dr Neneh Rowa-Dewar and Dr Sarah Wright for their guidance throughout this study.

\section{References}

1. Tavares W, Bowles R, Donelon B. Informing a Canadian paramedic profile: framing concepts, roles and crosscutting themes. BMC Health Serv Res 2016;16:1-16.

2. Donnelly $E$, Siebert $D$. Occupational risk factors in the emergency medical services. Prehosp Disaster Med 2009;24:422-9.

3. Donnelly EA, Bradford P, Davis M, Hedges C, Klingel M. Predictors of posttraumatic stress and preferred sources of social support among Canadian paramedics. CJEM 2016;18:205-12.

4. Halpern J, Gurevich M, Schwartz B, Brazeau P. Interventions for critical incident stress in emergency medical services: a qualitative study. Stress Health 2009a;25:139-49.

5. Drewitz-Chesney, C. Posttraumatic stress disorder among paramedics: exploring a new solution with occupational health nurses using the Ottawa Charter as a framework. Workplace Health and Safety. 2012;60:257-63.

6. Canadian Mental Health Association. 2019. Fast facts about Canadian mental illness. Available at: https://cmha.ca/fastfacts-about-mental-illness\#.Uw0Eo3lupg0

7. Carleton RN, Afifi TO, Turner S, et al. Mental disorder symptoms among public safety personnel in Canada. Can J Psychiatry 2018;63:54-64.

8. Regehr C, Goldberg G, Glancy GD, Knott T. Posttraumatic symptoms and disability in paramedics. ibid. 2002;47:953-8.

9. Petrie K, Milligan-Saville J, Gayed A, et al. Prevalence of PTSD and common mental disorders amongst ambulance personnel: a systematic review and meta-analysis. Soc Psychiatry Psychiatr Epidemiol 2002;53:897-909. https:// doi.org/10.1007/s00127-018-1539-5

10. Carleton RN, Afifi TO, Turner S, et al. Suicidal ideation, plans, and attempts among public safety personnel in Canada. Can Psychol 2018;59:220-31.

11. Halpern J, Gurevich M, Schwartz B, Brazeau P. What makes an incident critical for ambulance workers? Emotional outcomes and implications for intervention. Work Stress 2009b;23:173-89.

12. Haugen PT, McCrillis AM, Smid GE, Nijdam MJ. Mental health stigma and barriers to mental health care for first responders: a systematic review and meta-analysis. J Psychiatr Res 2017;94:218-29.

13. Koopmans E, Wagner SL, Schmidt G, Harder H. Emergency response services suicide: a crisis in Canada? J Loss Trauma 2017;22:527-39.

14. Hoyt T, Pasupathi M, Smith BW, et al. Disclosure of emotional events in groups at risk for posttraumatic stress disorder. Int J Stress Manag 2010;17:78-95.

15. Halpern J, Maunder RG, Schwartz B, Gurevich M. Identifying, describing, and expressing emotions after critical incidents in paramedics. J Trauma Stress 2012;25:111-4.

16. Lowery K, Stokes MA. Role of peer support and emotional expression on posttraumatic stress disorder in student paramedics. ibid. 2005;18:171-9.

17. Solomon Z, Mikulincer M. Life events and combat-related posttraumatic stress disorder: the intervening role of locus of control and social support. Mil Psychol 1990;2:241-56.

18. Marchand A, Boyer R, Nadeau C, Martin M. Predictors of posttraumatic stress disorders in police officers. Montreal, Quebec: The Institut de recherche Robert-Sauvé en santé et en sécurité du travail 2013. Available at: www.irsst.qc.cal media/documents/PubIRSST/R-786.pdf

19. Rimé B. Emotion elicits the social sharing of emotion: theory and empirical review. Emot Rev 2009;1:60-85. https://doi. org/10.1177/1754073908097189

20. Brewin CR, Andrews B, Valentine JD. Meta-analysis of risk factors for posttraumatic stress disorder in trauma-exposed adults. J Consult Clin Psychol 2000;68:748-66.

21. Ozer EJ, Best SR, Lipsey TL, Weiss DS. Predictors of posttraumatic stress disorder and symptoms in adults: a meta-analysis. Psychol Trauma 2008;S:3-36.

22.Prati G, Pietrantoni L. An application of the social support deterioration deterrence model to rescue workers. J Community Psychol 2010;38:901-17.

23. Charuvastra A, Cloitre M. Social bonds and posttraumatic stress disorder. Annu Rev Psychol 2009;59:301-28. https:// doi.org/10.1146/annurev.psych.58.110405.085650.Social

24.Preckel K, Kanske P, Singer T. On the interaction of social affect and cognition: empathy, compassion and theory of mind. Curr Opin Behav Sci 2018;19:1-6. https://doi. org/10.1016/j.cobeha.2017.07.010

25. Halpern J, Maunder RG, Schwartz B, Gurevich M. Downtime after critical incidents in emergency medical technicians/paramedics. Bio Med Res Int 2014;1-7.

26. Charmaz K. Constructing grounded theory: a practical guide through qualitative analysis. London: SAGE Publications Ltd, 2006.

27. Willis K, Daly J, Kealy M, et al. The essential role of social theory in qualitative public health research. Aust N Z J Public Health 2007;31:438-43. http://doi.org/10.1111/j.17536405.2007.00115.x 


\section{References (continued)}

28. BC Emergency Health Services. 2019. BC Ambulance Service - Who We Are. Available at: www.bcehs.ca/about/ who-we-are/bc-ambulance-service

29. Tracy S. Qualitative research methods: collecting evidence, crafting analysis, communicating impact. Chichester, West Sussex, UK: Wiley-Blackwell, 2013.

30. Malterud K, Siersma VD, Guassora AD. Sample size in qualitative interview studies: guided by information power. Qual Health Res 2016;26:1753-60.

31. Robinson OC. Sampling in interview-based qualitative research: a theoretical and practical guide. Qual Res Psychol 2014;11:25-41.

32. Williams A. Emotion work in paramedic practice: the implications for nurse educators. Nurse Educ Today 2012;32:368-72.

33. Santos JLG, Erdmann AL, de Sousa FGM, et al. Methodological perspectives in the use of grounded theory in nursing and health research. Anna Nery School Journal of Nursing 2016;20:1-8.

34. Groenewald T. Memos and memoing. In: Given LM, editor. The SAGE Encyclopedia of Qualitative Research Methods. Thousand Oaks: SAGE Publications Inc, 2008.

35. Saumure K, Given L. Rigor in qualitative research. In: Given LM, editor. The SAGE Encyclopedia of Qualitative Research Methods. SAGE Publications Ltd. 2012, p. 796.

36. Bowen GA. Supporting a grounded theory with an audit trail: an illustration. Int J Soc Res Methodol 2009;12:305-16.

37. Tufford L, Newman P. Bracketing in qualitative research. Qual Soc Work 2010;11:80-96.

38. Kennedy S, Kenny A, Meara PO. Student paramedic experience of transition into the workforce: a scoping review. Nurse Educ Today 2015;35:1037-43.

39. Jabr H. Retired police officers and emotional labour. 2011. MEd Thesis. Central Michigan University, Mount Pleasant Michigan.

40. Dwyer SC, Buckle JL. The space between: on being an insider-outsider in qualitative research. Int J Qual Methods 2009;8:54-63.

41.Pogrebin MR, Poole ED. Police and tragic events: the management of emotions. J Crim Justice 1991;19:395-403.

42. Erickson RJ, Grove WJC. Emotional labor and health care. Sociol Compass 2008;2:704-33.

43. Keating $C$. Why and how the silent self speaks volumes: functional approaches to nonverbal impression. In: Manusov V, Patterson ML, editors. The SAGE Handbook of Nonverbal Communication. Thousand Oaks: SAGE Publications Inc. 2006, p. 1-19.

44. Regehr C, Goldberg G, Hughes J. Exposure to human tragedy, empathy, and trauma in ambulance paramedics. Am J Orthopsychiatry 2002;72:505-13. https://doi. org/10.1037//0002-9432.72.4.505

45. Henckes N, Nurok M. The first pulse you take is your own - but don't forget your colleagues. Emotion teamwork in pre-hospital emergency medical services. Sociol Health IIIn 2015;37:1023-38. http://doi.org/10.1111/1467-9566.12261

46. Pruitt LD, Zoellner LA. The impact of social support: an analogue investigation of the aftermath of trauma exposure. J Anxiety Disord 2008;22:253-62.

47. Gouweloos-Trines J, Tyler MP, Giummarra MJ, et al. Perceived support at work after critical incidents and its relation to psychological distress: a survey among prehospital providers. Emerg Med J. 2017;34:816-22.

48. Howard C, Tuffin K, Stephens C. Unspeakable emotion: a discursive analysis of police talk about reactions to trauma. J Lang Soc Psychol 2000;19:295-314.

49. Stephens C, Long N. Communication with police supervisors and peers as a buffer of work-related traumatic stress. J Organ Behav 2000;21:407-24.

50. Holloway I. Qualitative research in health care. Open University Press, 2005.

51. Clompus SR, Albarran JW. Exploring the nature of resilience in paramedic practice: a psycho-social study. Int Emerg Nurs 2016;28:1-7. https://doi.org/10.1016/j. ienj.2015.11.006 